First publ. in: Chemical Physics Letters 382 (2003), 3-4, pp. 454-459

\title{
Fast electron dynamics in small aluminum clusters: non-magic behavior of a magic cluster
}

\author{
Peter Gerhardt, Marco Niemietz, Young Dok Kim *, Gerd Ganteför \\ Department of Physics, University of Konstanz, Universitaetsstrasse 10, D-78457 Konstanz, Germany
}

\begin{abstract}
Relaxation dynamics of the optically excited state in $\mathrm{Al}_{n}^{-}$with $n=6-15$ were studied using time-resolved two photon photoemission spectroscopy. Relaxation rates of these $\mathrm{Al}$ clusters are slower than those of some d-metal clusters which can be explained by lower electron density of state in the sp-metals. The relaxation rate of $\mathrm{Al}_{13}^{-}$, a magic cluster anion with a closed electronic shell is as fast as the non-magic clusters such as $\mathrm{Al}_{10}^{-}$, implying that the relaxation dynamics of magic clusters cannot be rationalized by the electron-electron scattering. Origin of this unexpected behaviour of the magic cluster is discussed.
\end{abstract}

Developments of experimental techniques using femtosecond (fs) lasers enabled obtaining information on relaxation dynamics of optically excited states (electron-hole excitation, or transient states in photochemical reactions) [1-11]. In solid states, the excess energy of the optically excited state is initially relaxed by electron-electron (e-e) scattering within a few tens of femtoseconds, whereas the conversion of this excess energy into the nuclear motion (e-phonon) takes place on the picosecond (ps) or nanosecond (ns) time scale. The relaxation of the optically excited state of nanomaterials compared to solids can be understood considering competition between transition and screening [12].

\footnotetext{
* Corresponding author. Fax: +49-7531-883888.

E-mail address: young.kim@uni-konstanz.de (Y. Dok Kim).
}

On one hand, the lower electron density in a nanocluster with respect to the bulk counterpart should increase the relaxation rate as a consequence of the reduced screening effect, as it was found for Ag nanoparticles [2]. On the other hand, discrete energy levels in a nanocluster lower the inelastic e-e scattering rate in comparison to that of the respective bulk metal with continuous unoccupied band above the Fermi level, which reduces the relaxation rate.

Recently, clusters consisting of d-metals such as $\mathrm{Ni}_{3}^{-}, \mathrm{Pd}_{3-7}^{-}$, and $\mathrm{Pt}_{3}^{-}$showed the relaxation rates of the optically excited states of only about 50-200 fs, which are comparable to the values for the respective bulk metals [13-16]. The energy separation between various sublevels in the valence Molecular Orbital (MO)s of these clusters is about $0.1 \mathrm{eV}$, and thus, numbers of unoccupied levels in 
these small clusters are large enough to allow the very fast e-e scattering [16]. For sp-metal clusters, much larger splittings of the MO schemes decrease the number of unoccupied levels within the excitation energy (pump photon energy) from the highest occupied molecular orbital (HOMO), increasing the relaxation times of the optically excited states by one or two orders of magnitude $\mathrm{Au}_{3}^{-}$: [17], $\mathrm{Na}_{2}$ : [18]. These previous results imply that for these small free mass-selected clusters, the relaxation dynamics is mainly governed by the auger-like e-e scattering, whereas the screening effects play a less important role. Note that only considering the screening effects, the lifetimes of the excited states in the sp-metal clusters with lower DOS should be increased with respect to those of the d-metal clusters, which does not reconcile the experimental data stated above. One can expect that the relaxation time in a sp-metal cluster becomes comparable to those of the d-metal clusters, when the cluster size is large enough, so that the separation between various energy levels becomes as narrow as those of the $\mathrm{Ni}_{3}^{-}, \mathrm{Pd}_{3-7}^{-}$, and $\mathrm{Pt}_{3}^{-}$clusters.

Of particular interest in cluster physics is the characterization of magic clusters. Magic clusters are chemically inert and exhibit closed shell structures [19] with relatively high electron affinities, and large HOMO-Lowest Unoccupied MO (LUMO) gaps. Well-known examples for the magic clusters are $\mathrm{C}_{60}$ and $\mathrm{Al}_{13}^{-}$[20-24]. Due to the high stabilities of these magic clusters, they are considered to be candidates for the building blocks of new cluster materials $[25,26]$. The relaxation times of the magic clusters are expected to be longer than those of other clusters due to lower numbers of the unoccupied states within the excitation energy above HOMO. In fact, $\mathrm{C}_{60}$ shows relaxation times in the order of tens of microseconds [27,28].

In this Letter, we present the Time-Resolved Two Photon Photoemission (TR-2PP) spectroscopy results taken for the mass-selected $\mathrm{Al}$ anion clusters consisting of 6-15 atoms in our new apparatus. We selected Al clusters, since the photon energy of our pump laser pulse is high enough to allow the excitation of the single electron-hole pair in $\mathrm{Al}_{13}^{-}$, which is a magic cluster with a gap of 1.5
eV [22-24]. Therefore, these studies enable a direct comparison of relaxation rates of the magic clusters with those of the non-magic counterparts. Surprisingly, $\mathrm{Al}_{13}^{-}$shows a similar relaxation rate to those of the non-magic clusters, i.e. the relaxation mechanism operating here seems to be independent on the electronic structure.

$\mathrm{Al}$ anion clusters are produced using a pulsed arc cluster ion source and mass-selected using a time-of-flight mass spectrometer [29]. A selected bunch of cluster anions is irradiated by two pulses generated by a femtosecond laser system [30]. The temperature of clusters is estimated to be room temperature [29]. The first pulse (pump pulse) has a photon energy of $1.55 \mathrm{eV}$ and the second pulse (probe pulse) a photon energy of $3.1 \mathrm{eV}$. The pump pulse results in photoexitation of a certain fraction of the anions in the bunch and the probe pulse detaches electrons from a fraction of the anions in the same bunch. The photon flux of both pulses has to be optimized to maximize the number of species hit by a single photon of each pulse, and to minimize the number of species hit by two or more photons from one of the pulses. For the pump/probe pulse alone, this has been achieved by adjusting the photon flux just below the onset of electron detachment by two-photon processes. The photon flux of both pump and probe pulses have to be readjusted for different clusters, because the cross sections of the corresponding processes may vary significantly as a function of cluster size.

In Fig. 1 a series of TR-2PP spectra for $\mathrm{Al}_{2}^{-}$ with various pump-probe delays is shown. For comparison, a standard Ultraviolet Photoelectron Spectrum (UPS) obtained with an excimer laser with $\mathrm{h} v=5.0 \mathrm{eV}$ is illustrated [31], in which an intense peak (A) can be observed at a binding energy of about $1.5 \mathrm{eV}$. In the TR-2PP spectra, a similar feature (A) can be observed with increased peak widths with respect to that in the standard UPS, due to the spectral width of the femtosecond pulse and Doppler effects caused by the higher kinetic energies of anions to increase the anion intensity. Switching-on of the pump pulse results in the appearance of a second feature (B) at a binding energy of $1.55 \pm 0.1 \mathrm{eV}$ lower than that of the peak $\mathrm{A}$. This feature can be assigned to nonresonant two-photon detachment of $\mathrm{Al}_{2}^{-}$. In a plot 


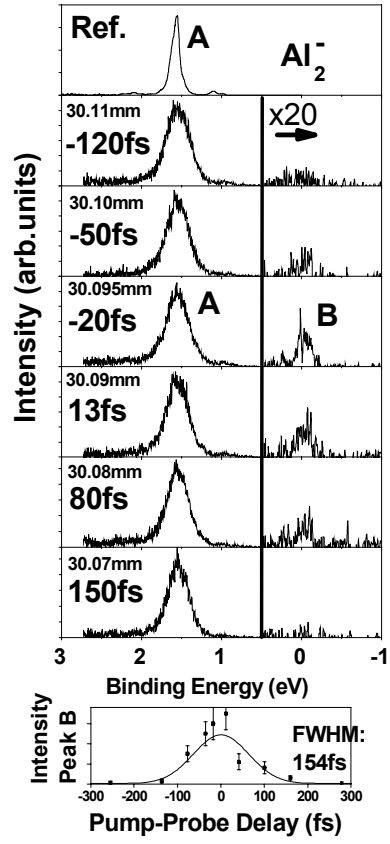

Fig. 1. Series of TR-2PP spectra of $\mathrm{Al}_{2}^{-}$. The photon energy of the pump pulse is $1.55 \mathrm{eV}$, and that of the probe pulse is $3.1 \mathrm{eV}$. The upper trace displays a standard spectrum recorded with an excimer laser $(h v=5.0 \mathrm{eV})$. The lower trace displays a plot of the intensity of feature B versus the shift of the retro-reflector. Time zero is to maximum intensity of feature B.

of the peak B intensity versus pump/probe time delay, which was fitted using a single gauss function, the Full Width of Half Maximum (FWHM) was determined to be $154 \mathrm{fs}$, which we attribute to the upper limit of our instrumental resolution. We define the time-delay for the maximum intensity of the peak B in Fig. 1 as zero-delay between pump and probe. The same procedure based on the nonresonant two-photon detachment signals of other species (e.g., $\mathrm{Al}_{3} \mathrm{O}^{-}$) also agrees well with the values aforementioned.

To shed light on relaxation dynamics of the mass-selected $\mathrm{Al}$ anion clusters, $\mathrm{Al}_{n}^{-}$for $n=6-15$ are investigated using TR-2PP spectroscopy. First, we focus on a non-magic cluster, $\mathrm{Al}_{10}^{-}$, which has a relatively high density of state above HOMO [22] (Fig. 2). A standard UPS spectrum of $\mathrm{Al}_{10}^{-}$obtained with an excimer laser $(h v=5.0 \mathrm{eV})$ is displayed on top of Fig. 2 [31]. In the series of TR2PP spectra in Fig. 2, a very broad shoulder

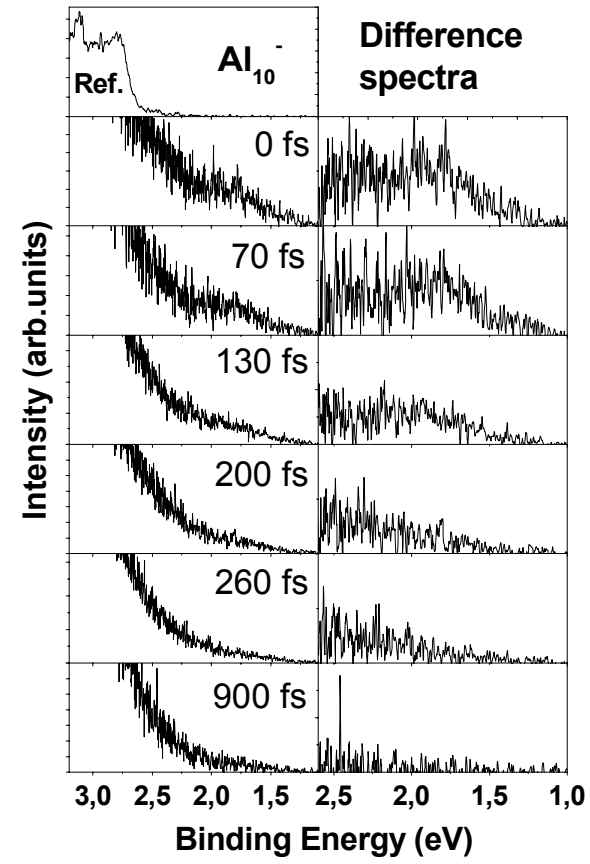

Fig. 2. Series of TR-2PP $(1.55 / 3.1 \mathrm{eV})$ photoelectron spectra of $\mathrm{Al}_{10}^{-}$. The top trace displays a standard UPS of $\mathrm{Al}_{10}^{-}$obtained with an excimer laser $(h v=5.0 \mathrm{eV})$ [33]. The TR-2PP spectra and the corresponding difference spectra are magnified to provide a better view of the binding energy regime between 2.5 and $1.0 \mathrm{eV}$, in which the true pump/probe signal is observed. A broad feature is visible between 1.5 and $2.0 \mathrm{eV}$ which disappears within $200 \mathrm{fs}$.

between 1.5 and $2 \mathrm{eV}$ appears which decreases in intensity with increasing delay time. This is obvious not only in the raw spectra but also in the difference spectra (Fig. 2). The signal shape develops (see $260 \mathrm{fs}$ ) into an exponential tale corresponding to a thermal distribution of the excitation energy within the electron gas. A similar behavior is found for transition metal clusters like $\mathrm{Ni}_{3}^{-}$[15]. At delay times larger than 900 fs no further change in the pump/probe spectra is observed. The still visible exponential tail corresponds to residual two photon processes of the probe pulse, and has to be subtracted to extract the true pump/probe signal (right side in Fig. 2). We assume that all excitation energy is dissipated into the vibrational degrees of freedom of the cluster when no further change of the pump/probe spectra is observed. The relaxation time can be 
determined from the spectra by plotting the intensity of the true pump/probe signal versus the delay time, which results in an exponential decrease [30]. For $\mathrm{Al}_{10}^{-}$, the relaxation time is estimated to be in the range of $200 \pm 70 \mathrm{fs}$. This time for the thermalization of $\mathrm{Al}_{10}^{-}$is about 2-3 times larger than the values observed for d-metal clusters due to the lower density of states in sp-metals. However, $200 \mathrm{fs}$ is still very fast which might be rationalized by relatively high density of state in the vicinity of $\mathrm{HOMO}$ for $\mathrm{Al}_{10}^{-}$, as mentioned above [22].

The TR-2PP spectra of all the $\mathrm{Al}_{n}^{-}$clusters are similar to the case of $\mathrm{Al}_{10}^{-}$except for $\mathrm{Al}_{13}^{-} . \mathrm{Al}_{13}^{-}$is a magic cluster with an icosahedral geometry, having 40 valence electrons forming a closed shell structure, and a HOMO-LUMO gap of $1.5 \mathrm{eV}$ [22-24,32]. Due to its high symmetry, the reference UPS spectrum of $\mathrm{Al}_{13}^{-}$recorded with $6.4 \mathrm{eV}$ photon energy shows a single peak at $3.8 \mathrm{eV}$ (Fig. 3, upper window, trace A). At a temperature of $300 \mathrm{~K}$, a single peak at $3.8 \mathrm{eV}$ is expected without any other features at lower binding energies, which has been already observed in the previous experiments $[22,23]$. However, in our reference spectrum, an additional broad tail can be observed between 2.5 and $3.5 \mathrm{eV}$. Comparing our reference spectrum with the UPS spectra of $\mathrm{Al}_{13}^{-}$for various temperatures in [23], our reference UPS spectrum in Fig. 3 consists of the 'hot' and 'room temperature' $\mathrm{Al}_{13}^{-}$ clusters, responsible for the broad tails between 2.5 and $3.5 \mathrm{eV}$ and a distinct peak at $3.8 \mathrm{eV}$, respectively [23]. We assign the tail at lower binding energies to two-photon processes. The energy of the first photon is thermalized within the duration of a nanosecond pulse and the second photon detaches an electron from this 'hot' cluster.

The relaxation time of the $\mathrm{Al}_{13}^{-}$is almost same as that of $\mathrm{Al}_{10}^{-}$(Fig. 3). However, in contrast to all other difference spectra a narrow peak (Peak B in Fig. 3) appears at a binding energy corresponding to the LUMO overlapped with a broad background signal in the energy range between 1.5 and $3 \mathrm{eV}$. The appearance of the peak B in the pump/ probe signal can be explained by a standard pump/ probe-event (single photon contribution from each pulse), i.e. the pump pulse $(h v=1.55 \mathrm{eV})$ excites one electron from the HOMO to the LUMO, and

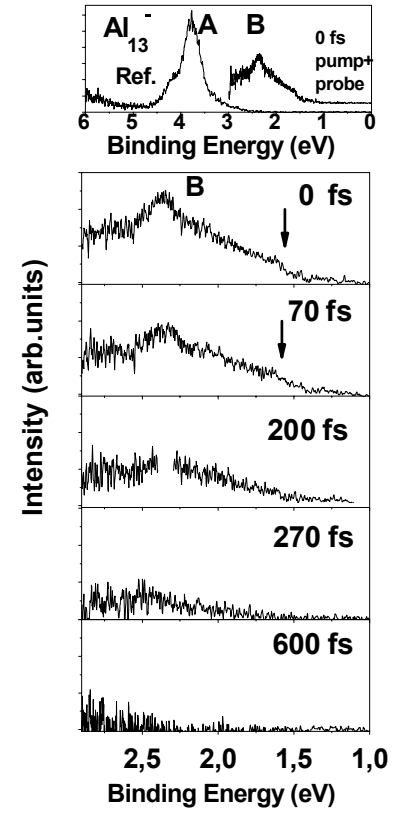

Fig. 3. TR-2PP difference spectra of $\mathrm{Al}_{13}^{-}$. The first spectrum from the top (trace A) is standard UPS spectrum obtained with an excimer laser $(h v=6.4 \mathrm{eV})$. The series of five spectra displays the time-dependence of the pump-probe signal. The pumpprobe spectrum recorded at zero delay is also displayed in the top window for the comparison. The observation of a narrow peak (marked B) in the TR-2PP spectra is unique for the $\mathrm{Al}_{n}^{-}$ clusters with $n=6-15$. The broad 'background'-like signal between $1.5-3 \mathrm{eV}$ is attributed to multiphoton processes (see text).

a probe pulse $(h v=3.1 \mathrm{eV})$ detaches the excited electron. Electrons detached by this process appear at a kinetic energy of $0.85 \mathrm{eV}$ (pump photon (1.55 $\mathrm{eV})+$ probe photon $(3.1 \mathrm{eV})$ - binding energy of HOMO $(3.8 \mathrm{eV}))$ corresponding to a binding energy of $2.15 \mathrm{eV}$ (peak B). The broad background signal can be explained by a two-photon event of the probe pulse. Similar background features are observed even when the pump pulse is switchedoff, giving rise to an electron signal (not shown) extending up to a maximum kinetic energy of 2.4 $\mathrm{eV}(2 \times 3.1-3.8 \mathrm{eV}$, binding energy $0.6 \mathrm{eV})$. It is noteworthy that only broad features can be observed up to the binding energy of $0.6 \mathrm{eV}$ without any distinct peaks. One possible explanation for this result is the electron-phonon scattering which might be sufficiently fast to allow thermalization of the energy of the first photon within the duration of a probe pulse. The 'background' signal visible in 
the difference spectra of Fig. 3 can be caused by a process involving contributions of one photon from the pump pulse and two photons from the probe pulse. The assignment is supported by the observation of the small step-like structure (see arrows in Fig. 3) at a binding energy of about 1.6 $\mathrm{eV}$. We assign this feature to two-photon $(2 \times 3.1$ $\mathrm{eV}$ ) transitions from anions excited by absorbing a pump photon $(1.55 \mathrm{eV})$ into the excited state of neutral $\mathrm{Al}_{13}$. The energy of the excited state of the neutral $\mathrm{Al}_{13}$ is higher than that of the neutral ground state by about $2.5 \mathrm{eV}$. The kinetic energy of the electrons detached via this process $(2 \times 3.1+1.55 \mathrm{eV} 7-3.8-2.5 \mathrm{eV})$ should be 1.45 $\mathrm{eV}$, corresponding to a binding energy of $1.65 \mathrm{eV}$. (The energy of the excited state of the neutral cluster in the geometry of the anion is extracted from the photoelectron spectrum of the anion).

The relaxation times for $\mathrm{Al}_{n}^{-}$with $n=6-15$ are compared in Fig. 4, which shows that the relaxation rate of $\mathrm{Al}_{13}^{-}$is comparable to the other nonmagic clusters. One may argue that there is no defined geometric structure and no gap for $\mathrm{Al}_{13}^{-}$ due to extremely high cluster temperatures [23]. However, existence of the clusters with such an increased temperature is unlikely in our experimental set-up [29]. In this context, note that we recently determined the relaxation time of $\mathrm{Au}_{6}^{-}$, which has a large HOMO-LUMO gap in the neutral state, to be longer than $1 \mathrm{~ns}$ using the same

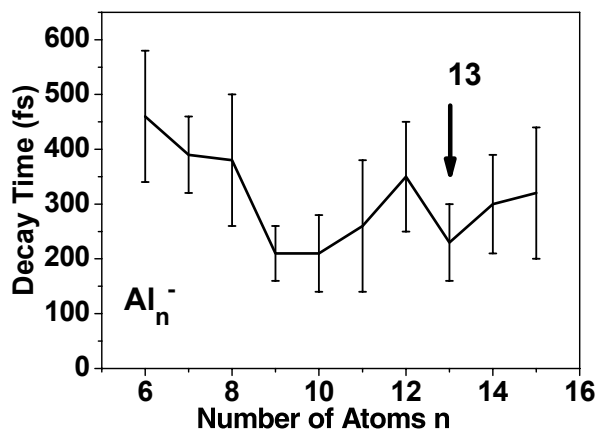

Fig. 4. Relaxation times of the $\mathrm{Al}_{n}^{-}(n=6-15)$ corresponding to the exponential decay of the TR-2PP signals with increasing pump/probe delay. The error bars correspond to the uncertainty in the determination of the intensities of the true pump/ probe signals with respect to the background signals originating from multi-photon processes. experimental set-up [33], implying that the cluster temperature of the magic clusters are low enough to ensure defined geometries. We also exclude contributions from collective excitations, since in $\mathrm{Al}$, the bulk and surface plasmons are at much higher energies (above $10 \mathrm{eV}$ ) [34].

We should come up to a conclusion that the relaxation mechanism should involve a process, which occurs for Al but not for Au. Moreover, the relaxation dynamics should be independent on the detailed electronic structure of the cluster. One possible explanation might be a very fast electronphonon interaction. Indications for a faster relaxation route for bulk $\mathrm{Al}$ than those of other metals have been already reported in previous studies [35]. In clusters, effects like a 'fluxional' geometry induced by the lower coordinated 'surface' atoms might yield a much stronger electronphonon coupling than in the crystalline bulk. To better understand the relaxation dynamics of magic clusters, further theoretical as well as experimental studies are warranted.

In conclusion, we have presented the relaxation dynamics of $\mathrm{Al}_{n}^{-}$with $n$ from 6 to 15. The relaxation rates of non-magic $\mathrm{Al}$ clusters such as $\mathrm{Al}_{10}^{-}$are 2-3 times slower than those of $\mathrm{Pt}_{3}^{-}, \mathrm{Pd}_{3-7}^{-}$[13-15], but still as fast as that of $\mathrm{Ni}_{3}^{-}$[10]. The relaxation rate of $\mathrm{Al}_{13}^{-}$, a magic cluster with a large HOMOLUMO gap $(1.5 \mathrm{eV})$, was found to be as fast as other non-magic clusters, indicating the existence of a very effective relaxation mechanism, which is independent on the electronic structure.

\section{Acknowledgements}

We gratefully acknowledge the financial supports from DFG (Deutsche Forschungsgemeinschaft).

\section{References}

[1] A.H. Zewail, in: Femtochemistry, vol. I and II, World Scientific, Singapore, 1994.

[2] C. Voisin, D. Christofilos, N.D. Fatti, F. Vallee, B. Prevel, E. Cottancin, J. Lerme, M. Pellarin, M. Broyer, Phys. Rev. Lett. 85 (2000) 2200. 
[3] D.H. Paik, N.J. Kim, A.H. Zewail, J. Chem. Phys. 118 (2003) 6923.

[4] J.M. Weber, J. Kim, E.A. Woronowicz, C.H. Weddle, I. Becker, O. Cheshnovsky, M.A. Johnson, Chem. Phys. Lett. 339 (2001) 337.

[5] S. Vajda, C. Lupulescu, A. Merli, F. Budzyn, L. Wöste, M. Hartmann, J. Pittner, V. Bonacic-Koutecky, Phys. Rev. Lett. 89 (2002) 213404.

[6] W.L. Lineberger, P. Wenthold, D. Hrovat, W.T. Borden, Science 272 (1996) 1456.

[7] R. Schlipper, R. Kusche, B. von Issendorf, H. Haberland, Phys. Rev. Lett. 80 (1998) 1194.

[8] H. Petek, S. Ogawa, Prog. Surf. Sci. 56 (1997) 239.

[9] E. Knoesel, A. Hotzel, T. Hertel, M. Wolf, G. Ertl, Surf. Sci. 368 (1996) 76.

[10] T. Hertel, E. Knoesel, M. Wolf, G. Ertl, Phys. Rev. Lett. 76 (1996) 535.

[11] W.S. Fan, R. Storz, H.W.K. Tom, J. Boker, Phys. Rev. B 46 (1992) 13592.

[12] P.M. Echenique, J.M. Pitarke, E.V. Chulkov, A. Rubio, Chem. Phys. 251 (2000) 1.

[13] N. Pontius, P.S. Bechthold, M. Neeb, W. Eberhardt, Phys. Rev. Lett. 84 (2000) 1132.

[14] N. Pontius, G. Lüttgens, P.S. Bechthold, M. Neeb, W. Eberhardt, J. Chem. Phys. 115 (2001) 10479.

[15] N. Pontius, P.S. Bechthold, M. Neeb, W. Eberhardt, J. Elec. Spec. Relat. Phenom. 114-116 (2001) 163.

[16] N. Pontinus, M. Neeb, W. Eberhardt, G. Lüttgens, P.S. Bechthold, Phys. Rev. B 67 (2003) 035425.

[17] G. Ganteför, S. Kraus, W. Eberhardt, J. Elec. Spec. Relat. Phenom. 88-91 (1998) 35.

[18] A. Assion, M. Geisler, J. Helbing, V. Seyfried, T. Baumert, Phys. Rev. A 54 (1996) R4605.
[19] W.D. Knight, K. Clemenger, W.A. de Herr, W.A. Saunders, M.Y. Chou, M.L. Cohen, Phys. Rev. Lett. 52 (1984) 2141.

[20] H.W. Kroto, J.R. Heath, S.C. O'Brien, R.F. Curl, R.E. Smalley, Nature (London) 318 (1985) 162.

[21] S.H. Yang, C.L. Pettuette, J. Conceicao, O. Cheshnovsky, R.E. Smalley, Chem. Phys. Lett. 139 (1987) 233.

[22] X. Li, H. Wu, X.-B. Wang, L.-S. Wang, Phys. Rev. Lett. 81 (1998) 1909.

[23] J. Akola, M. Manninen, H. Häkkinen, U. Landman, X. Li, L.-S. Wang, Phys. Rev. B 60 (1999) R11297.

[24] S. Buckart, N. Blessing, B. Klipps, J. Müller, G. Ganteför, G. Seifert, Chem. Phys. Lett. 301 (1999) 546.

[25] S.N. Khanna, P. Jena, Phys. Rev. Lett. 69 (1992) 1664.

[26] U. Röthlisberger, W. Andreoni, M. Parrinello, Phys. Rev. Lett. 72 (1994) 665.

[27] J.W. Arbogast, A.P. Darmanyan, C.S. Foote, Y. Rubin, F.N. Diederich, M.M. Alvarez, S.J. Anz, R.L. Whetten, J. Chem. Phys. 95 (1995) 11.

[28] R.W. Haufler, L.-S. Wang, L.P.F. Chinabte, C. Jin, J. Conceicao, Y. Chai, R.E. Smalley, Chem. Phys. Lett. 179 (1991) 449.

[29] C.-Y. Cha, G. Ganteför, W. Eberhardt, Rev. Sci. Instrum. 63 (1992) 5661.

[30] S. Minemoto, J. Müller, G. Ganteför, H.J. Münzer, J. Boneberg, P. Leiderer, Phys. Rev. Lett. 84 (2000) 3554.

[31] C.-Y. Cha, G. Ganteför, W. Eberhardt, J. Chem. Phys. 100 (1994) 1.

[32] S.N. Khanna, P. Jena, Chem. Phys. Lett. 218 (1994) 383.

[33] Marco Niemietz, Peter Gerhardt, Gerd Ganteför, Young Dok Kim, Chem. Phys. Lett. 380 (2003) 99.

[34] M. Rocca, Surf. Sci. Rep. 22 (1995) 1.

[35] M. Bauer, S. Pawlik, M. Aeschlimann, Proc. of the SPIE 3272 (1998) 201. 